\title{
Airway management of patients undergoing laparoscopic gastric bypass surgery: a single center analysis
}

\author{
Sung An Kang, Gun Woo Kim, Yeo Sam Yoon, Choon Soo Lee, and Jang-Ho Song \\ Department of Anesthesiology and Pain Medicine, Inha University College of Medicine, Incheon, Korea
}

Gastric bypass surgery is recognized as the most effective treatment for severely obese patients who cannot effectively control their weight through diet and exercise. During gastric bypass surgery, proper anesthetic management requires special attention from preoperative assessment to postoperative recovery, especially for airway management.

We reviewed the electronic medical records of 66 patients who received laparoscopic gastric bypass surgery for being overweight or diagnosed as obesity from January 2011 to May 2013 and analyzed the collected data in relation to the body mass index (BMI) as follows: Group 1 - BMI of 25 to less than 30 ( $\mathrm{n}=$ 5); Group 2 - BMI of 30 to less than $35(\mathrm{n}=12)$; Group 3 - BMI of 35 to less than $40(n=2)$; Group 4 - BMI of 40 to less than $50(\mathrm{n}=16)$; and Group 5 - BMI of 50 or greater $(\mathrm{n}=5)$. Among the subject patients, $78.8 \%$ had metabolic syndrome, $48.5 \%$ had diabetes, $43.9 \%$ had hypertension, $33.3 \%$ had both diabetes and hypertension. The prevalence rates of metabolic syndrome, type 2 diabetes mellitus, and hypertension were not significantly different among the groups. Polysomnography (PSG) performed on 60 of the 66 subject patients revealed that 41 patients (68.3\%) had moderate to severe obstructive sleep apnea (OSA, Apneahypopnea index; AHI of 15 or greater). Laryngoscopic view during intubation showed $97 \%$ of the patients with grade 1 or $2,3 \%$ with grade 3 and $0 \%$ with grade 4 (Grade 1: no difficulty in laryngoscopic view, Grade 2: only posterior extremity of the glottis is visible, Grade 3: only the epiglottis is seen, and Grade 4: no recognizable structures). There were no significant inter-group differences in the number of patients with difficult intubations corresponding to grade 3 or 4 . During the induction of patients undergoing gastric bypass surgery, difficult airways or intubation should be anticipated. Therefore, it is very important to prepare airway management devices, including a surgical airway. Brodsky et al. reported that in morbid obese patients, the Mallampati score and neck circumference were more precise predictable factors in determining difficult intubation rather than weight and body mass index [1]. Using the ramped position, in which the external acoustic meatus and sternal notch heights are matched, provides a higher success rate in the intubation of severe obese patients compared to the standard sniffing position. All subject patients in our analysis received intubation in the ramped position. During surgery, the number of positive end expiratory pressure (PEEP) applied patients for the 5 groups were $0(0 \%), 3$ (25.0\%), 17 (60.7\%), 13 (81.3\%) and 4 (80.0\%), respectively. The number of patients admitted to the intensive care unit immediately after surgery for each of the 5 groups were 1 (20.0\%), 1 (8.3\%), $1(3.6 \%), 1(6.3 \%)$ and $2(40.0 \%)$, respectively, and there were no significant differences among the groups.

Obese patients who undergo gastric bypass surgery have a relatively high incidence of obstructive OSA. OSA is the occurrence of apnea or hypopnea due to repetitive partial or complete blockade of the upper airway. Patients with OSA have a higher risk of airway obstruction after general anesthesia and they are more susceptible to myocardial ischemia, congestive heart failure, cerebrovascular ischemia, and hypoxemia [2]. This risk is not only due to the residual effects of the anesthetic agents including muscle relaxants and opioids but also due to a decrease in the upper airway muscle tone. While proper diagnosis and management of OSA prior to gastric bypass surgery will be

Corresponding author: Jang-Ho Song, M.D., Department of Anesthesiology and Pain Medicine, Inha University College of Medicine, Inha University Hospital, 27, Inhang-ro, Jung-gu, Incheon 400-711, Korea. Tel: 82-32-890-3937, Fax: 82-32-890-3900, E-mail: jhs@inha.ac.kr (c) This is an open-access article distributed under the terms of the Creative Commons Attribution Non-Commercial License (http:// creativecommons.org/licenses/by-nc/3.0/), which permits unrestricted non-commercial use, distribution, and reproduction in any medium, provided the original work is properly cited. 
helpful in reducing the above risks, perioperative application of continuous positive airway pressure (CPAP) has been regarded to reduce the incidences of postoperative complications in OSA patients undergoing gastric bypass surgery [3]. In our analysis, for subject patients with an AHI of 15 or greater, CPAP to the appropriate degree was administered before and after surgery.

In obese patients, due to the occurrence of atelectasis, abnormal pulmonary gas exchange occurs at a higher rate compared to normal people during anesthesia for laparoscopic surgery. In order to overcome this, application of PEEP has been reported to be an effective method [4].

This analysis at a single university hospital showed that patients undergoing gastric bypass surgery had higher incidences of OSA and required anesthesia management consisting of CPAP, intubation in the ramped position, and the application of PEEP.

\section{References}

1. Brodsky JB, Lemmens HJ, Brock-Utne JG, Vierra M, Saidman LJ. Morbid obesity and tracheal intubation. Anesth Analg 2002; 94: 732-6.

2. Vasu TS, Grewal R, Doghramji K. Obstructive sleep apnea syndrome and perioperative complications: a systematic review of the literature. J Clin Sleep Med 2012; 8: 199-207.

3. Weingarten TN, Flores AS, McKenzie JA, Nguyen LT, Robinson WB, Kinney TM, et al. Obstructive sleep apnoea and perioperative complications in bariatric patients. Br J Anaesth 2011; 106: 131-9.

4. Pelosi P, Ravagnan I, Giurati G, Panigada M, Bottino N, Tredici S, et al. Positive end-expiratory pressure improves respiratory function in obese but not in normal subjects during anesthesia and paralysis. Anesthesiology 1999; 91: 1221-31. 